\title{
Predicate the Ability of Extracorporeal Shock Wave Lithotripsy (ESWL) to treat the Kidney Stones by used Combined Classifier
}

\author{
Samera Shams Hussein Lubab Ahmed Tawfeeq Sukaina Sh Altyar \\ Department of Computer Science, College of Education for Pure \\ Science, Baghdad University \\ sameramazn@yahoo.com \\ Sukaina_altyar@yahoo.com
}

Recived : 271112018

Revised : 2\12\2018

Available online : $\quad 25 / 1 / 2019$

DOI: $10.29304 / j q c m .2019 .11 .1 .466$

\begin{abstract}
:
Extracorporeal Shock Wave Lithotripsy (ESWL) is the most commonplace remedy for kidney stone. Shock waves from outside the body frame are centered at a kidney stone inflicting the stone to fragment. The success of the (ESWL) treatment is based on some variables such as age, sex, stone quantity stone period and so on. Thus, the prediction the success of remedy by this method is so important for professionals to make a decision to continue using (ESWL) or to using another remedy technique. In this study, a prediction system for (ESWL) treatment by used three techniques of mixing classifiers, which is Product Rule (PR), Neural Network (NN) and the proposed classifier called Nested Combined Classifier (NCC). The samples had been taken from 2850 actual sufferers cases that had been treated at Urology and Nephrology center of Iraq. The results from three cases have been compared to actual treatment results of (ESWL) for trained and nontrained cases and compared the results of three models. The results show that (NCC) approach is the most accurate method in prediction the efficient of uses (ESWL) remedy in treatment the kidney stone.
\end{abstract}

Keywords: Extracorporeal Shock Wave Lithotripsy, Product Rule, Neural Network, ANN, PR. 


\section{Introduction:}

Extracorporeal shock wave lithotripsy (ESWL) is a nonsurgical technique that makes use of highenergy surprise waves to interrupt a kidney stone into "stone dirt" or fragments that could more easily travel via the urinary tract and skip from the frame. ESWL was added into scientific practice within the 1980s, and due to the fact that then has become one of the principal treatment alternatives in patients with renal and/or ureteral calculi. but, the development of endourology and minimally invasive surgical procedures with their excessive achievement fees has reduced its applicability. From then on, it has emerged as necessary to search for the foremost technical parameters and careful selection of candidates for ESWL on the way to optimize its consequences and justify its indication. [1].

ESWL goes better with some stones than the others. Very big stones are unable to be treated by this technique. The shape and size of stone, wherein it is lodged inside urinary tract, patient health and his kidneys' health will probably be aspect of the decision to use it. Stones which are lower than $2 \mathrm{~cm}$ in diameter are the ideal size for SWL. The treatment with SWL probably won't be successful in very big ones. SWL is more suitable for some people rather than others. Considering that shock waves and X-rays are required in SWL, pregnant women that have stones will not be treated by this way. People with severe skeletal abnormalities, infections, bleeding disorders, or who are morbidly obese also not commonly good candidates for SWL. In case patient kidneys have any other abnormalities, the doctor may possibly decide that the patient need to use another treatment. If patient come with a cardiac pacemaker, the heart specialist will decide if patient can be treated by ESWL [2].

A perfect estimation of the probability to eliminate the stone from individual's kidney are required for appropriate treatment choice to figure out who will have optimum benefit from ESWL. Thus, to identity the prognostication factors that effect on clearing away stone from kidney by utilizing ESWL will be uses for predication result of treatment via utilized artificial intelligence techniques [3].
A range of computer models was developed in the field of machine learning and statistics which could be used for predicting medical results, such as decision trees, logistic regression (LR), Bayesian networks and artificial neural networks (ANNs). Perhaps the best commonly used methods are depending upon the statistical technique of regression. For researches with a binary endpoint (for example, yes/no, alive/ dead), the LR is used usually. For the testing of time to event data, the Cox proportional hazards regression is the standard. These methods are becoming standard because of their relative simplicity, the widely used availability of pc software to meet these models, the inference that permit by evaluate the fitted model coefficients, and the achieved statistical theory which supplements and supports their use [4]. During the last decade, a unique class of techniques called artificial neural networks (ANNs) have been proposed to be the alternative or supplement to standard statistical techniques. Artificial neural network is an important part of artificial intelligence which offer an "intelligent" method of predicting practical outcomes with higher efficiency and accuracy. ANN algorithm is dependent on the idealized design of a biological neuron (unit) and presents very good promise in conquering the complexities in actions of biosystems/ materials that are otherwise hard to comprehend. Therefore, ANNs could be played as a model of human brain function, in which sets of data in the sort of input and output patterns are organized to train the ANN. The ANN classifiers can also be enhancing via combining their back class estimates with conventional language model likelihood ratios, by using a logistic regression combiner [5].

The aim of this study is to utilize a Nested Combined Classifiers (NCC), a method of combination in the classification area, hoping to increase the accuracy of classification in area of predication treatment the kidney stone by ESWL. NCC combined the results of combination using neural networks and the results of combination using product rule. 
This study applied the following techniques in classification:

- Combination using Neural Networks.

- Combination using Product Rule.

- $\quad$ Nested Combination by Combination 1, 2.

\section{Previous Works}

ANN and LR have been utilized in a variety of domains in medical diagnosis. Currently, ANN have been applied for estimating risk in a wide range of application such as breast cancers. In other hand, LR has been utilized for estimation the disease risk in prostate cancer, breast cancer, coronary heart disease, postoperative complications), and stroke.

Hamid et al (2003) [6], had examine the ability of ANN to predict perfect renal stone fragmentation in people getting treatment by ESWL. The research used 82 patient's cases that have renal stones which they had been treated by ESWL. For training process, they used 60 patient's cases that got most effective fragmentation of stones by utilized ESWL. These data generally involved the settings of ESWL that been used, the $24 \mathrm{~h}$ urinary variables, and the stone disease radiological features. The predication accuracy was tested on 22 non-trained patients, by providing the input parameters of the 22 patients towards the trained ANN and acquiring the predicted values. The tested results prove that the trained ANN forecast the optimum fragmentation in $\leq 13 \quad 000$ shocks/stone in 17 patients and optimum fragmentation in>13 000 shocks/stone in the other 5 patients. The total correlation among the observed and predicted values was $75.5 \%$ in these 17 patients.

Goyal et al (2010) [7], compared the accuracy of multivariate regression analysis and ANN analysis for fragmentation of renal stone by ESWL. 276 total patients with renal calculus had been treated by ESWL at the time of (December 2001) to month of December in year of 2006.
Of those, the 196 patient's cases have been used to build data that had been used for training the ANN. The predication accuracy of trained ANN was tested on 80 non-trained patients. The input data involve patient age, stone burden and size, urinary $\mathrm{pH}$ and number of sittings. For nontrained 80 patients, the input was examined and result had also determined by MVRA. The predicted value from both the methods had been compared and the results had been sketched. The observed and predicted number of shocks and values of shock power had been compared using 1:1 slope line. The results had been computed as coefficient of correlation. In summary, ANN gives better coefficient of correlation than MVRA, therefore is seen as a better tool to evaluate the perfect renal stone fragmentation by way of ESWL.

Seckiner et al (2010) [8], developed an artificial neural network model by making use of data from patients that have renal stone, to be able to predict stone-free situation and to make it possible for identifying treatment with ESWL for renal stones. The data had been collected from the 203 patients involves age, gender, stone size and density, stone size after ESWL, location of the stone, skin to stone distance, stone nature (single or multiple), and some other parameters. ANN method and regression analysis had been applied to estimate treatment success utilizing the same series of data. The consequently, patients had been divided into three groups by ANN software, to be able to implement the ANN which are: $\mathrm{n}=139$ training group, $\mathrm{n}=32$ validation group, and $\mathrm{n}=32$ test group. The results show that the accuracy of the free stone rate was $99.25 \%$ in the training group, and it achieved $85.48 \%$ in the validation group, and it got $88.70 \%$ in the test group. 


\section{Theoretical Background}

\section{Logistic Regression (LR)}

This method is inspects the relationship in between the binary outcome variable (dependent) like absence or presence of disease and predictor (independent or explanatory) variables like imaging findings or demographics of patient. For example, the absence or presence of breast cancer within a certain time period would possibly be predicted from information of the patient's breast density, age, genealogy and family history of breast cancer, and any previous breast procedures. The outcomes variables could be both categoric and continuous. If $\mathrm{X} 1, \mathrm{X} 2, \mathrm{X} 3$ to $\mathrm{Xn}$ denote $\mathrm{n}$ predictor variables (for example, calcification types, patient age, breast density, etc,), $\mathrm{p}$ denotes the possibility of disease existence, the equation defines the relationship between $\mathrm{p}$ and the predictor variables is given by [9]:

$$
\begin{gathered}
\log \left(\frac{p}{1-p}\right)=\beta_{0}+\beta_{1} X_{1}+ \\
\beta_{2} X_{2}+\cdots+\beta_{n} X_{n}
\end{gathered}
$$

Where: $\beta_{0}$ is a constant and $\beta_{1}, \beta_{2}, \ldots, \beta_{n}$ represent the regression coefficients for the predictor variables $X_{1}, \ldots, X_{n}$.

The regression coefficients $\beta$ can be calculated from available data. Every regression coefficient represents the contribution size of the related predictor variable for the outcome.

The effects of the predictor variables on the output variable is generally determined by utilizing the odd ratio of the predictor variable that represents the factor wherein the odds of an outcome adjust for a one-unit modify in the predictor variable. The odds ratio can be estimated through taking the exponential of the coefficient (for example, exp $\left(\beta_{1}\right)$. As an example, if $\beta_{1}$ is the coefficient of $\mathrm{XFH}$ variable (which represents the family history of breast cancer), and $\mathrm{p}$ presents the breast cancer probability, then the $\exp \left(\beta_{1}\right)$ is the odds ratio related to the family history of breast cancer.
In such a case, the odds ratio presents the factor where the odds of featuring breast cancer raise if the family of patient has a history of breast cancer and almost all remaining predictor variables keep unchanged. This means that, if the odds ratio related to the family history of patient with breast cancer is 2 , hence the breast cancer may happen twice in women that have a family history of breast cancer than the women with no such family history. LR models usually involve only the variables which can be considered "important" in predicting the outcome. Through use of $\mathrm{P}$ values, the variables importance is described in relation to of the statistical value of the variables coefficients. The significance criterion $\mathrm{P} \leq 0.05$ is generally used whenever testing for the statistical significance of variables; nevertheless, these types of criteria can vary based on the quantity of available data. As an example, if the observations number is very big, predictors with little effects on the outcome could also become significant. THE various techniques can yield a variety of regression models, that they generally work similarly. Often, medically important variables could be found to be statistically insignificant through the selection methods due to the fact their influence might be attenuated by the existence of other strong predictors. In these cases, these medically important variables can however be involved in the model regardless of their statistical significance level [9].

\section{Neural Network}

ANN is a computer models stimulated the system of biologic neural networks that is a part of the machine learning techniques in order to solve the complicated nonlinear systems in the realistic life. ANN have been widely used in numerous research areas ranging from marketing to medicine. Generally, in most cases the neural network is an adaptive system which modifies its structure throughout a learning phase. ANN can learn and identify correlated pattern between inputs and related outputs [10]. Fig. 1 illustrates a basic example of an ANN. 


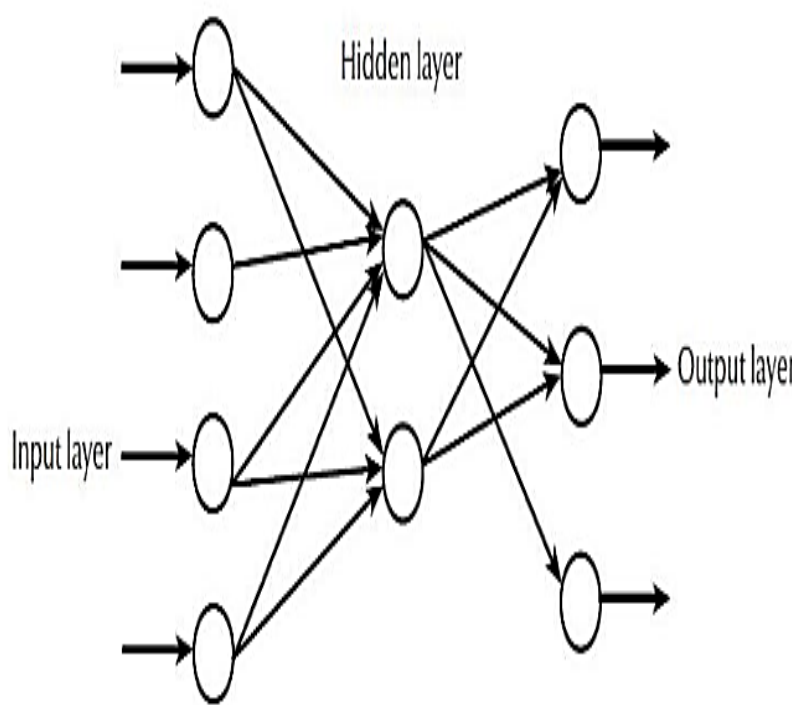

Figure 1: Typical structure of three-layer ANN which have four neurons in the input layer, two neurons in the hidden layer and three neurons in the output layer, without having direct connection from input layer to output layer [10]

As shown in figure 1, the interconnections don't loop back or skip any other neurons, this type of network is called feedforward [9]. In these networks, there are two functions concerning the behavior of a unit in a specific layer and influence the generalization of the model. One of those is input function and the second one is output function that is often known as the activation function. The equation of input function is given by [10]:

$$
y=x_{1} w_{1}+\cdots,+x_{n} w_{n}+b
$$

Where $n$ is the patterns number in the data set, $x$ is the data points, which are called input variables or features with identified class memberships.Many non-linear functions have been used, and the most popular one is sigmoid function, because it is able to show both linear and non-linear property. The sigmoid function is given by Eq. (3) [10],

$$
f(x)=\frac{1}{1=e^{-x}}
$$

ANN are becoming highly popular with data mining practitioners, especially in marketing, finance and medical research. It is because they provide the major advantage of not being dependent on "a priori" assumptions and of enabling detection of links amongst factors that conventional statistical methods like LR might not be able to detect [10]. Comparing artificial neural network models with linear models of standard statistical generalized like LR is a significant step in the development method [11]. In case the results reveal that the gain of using a nonlinear model, like the ANN, is limited, then it should often choose the less complicated model.

\section{Receiver Operating Characteristic (ROC) \\ Curves}

ROC curves is the method used to determine the predictive utility via displaying the trade-off between the false-positive rate and the truepositive rate that inherent in finding specific thresholds where predictions may be based. The area below this curve presents the likelihood that provided a negative and positive case, the output of classifier is going to be higher for the positive case and it isn't depending on the choosing of decision threshold. Using this method is less dependent on the malignancy frequency in the population and permits considering the and specificity of the model and the sensitivity at several probability levels. An effective one-class classifier should have both a mini fraction false negative as a mini fraction false positive. However, the ROC curve provides a very good summary of the efficiency of a one-class classifier, it is actually difficult to compare two ROC curves. The best way to summarize a ROC-curve with a single number often is the Area below the ROC Curve. This integrates the fraction false positive around ranging thresholds (or equivalently, ranging fraction false negative). Lesser values mean a better separation between out layer objects and the target. The graph in Figure 3 shows 3 ROC curves which represent excellent, and useless tests plotted on a single graph [11]. 


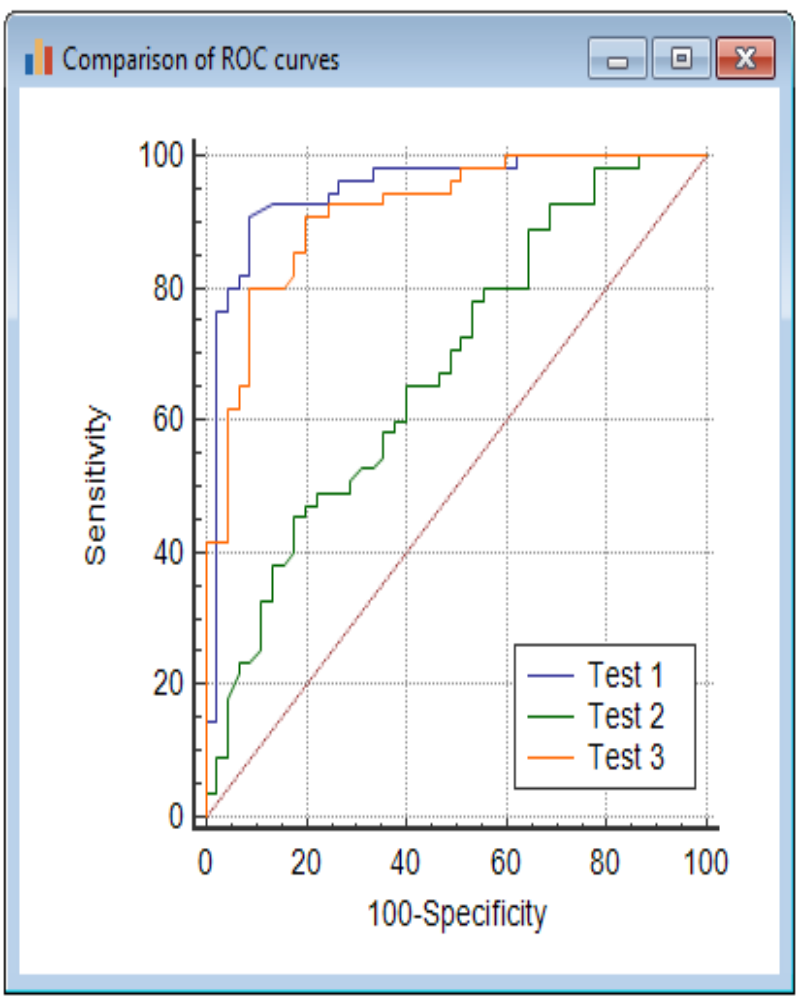

Figure 2: Comparing ROC Curves. Test 1 (Green) Worthless, Test 2(Orange) Good, and Test 3 (green) Excellent

The accuracy of the test is depending on how the approach separates the group that tested into those can and cannot treated by SWL. The Accuracy is measured via the area under the ROC curve.

\section{The Proposed Approaches}

Three approaches of hybrid classification rules have been proposed. The first approach is "Product Rule" termed (PR) which is based on combined neural network (NN) then logistic regression (LR) and the second approach is termed (NN1) which is adding outputs of the statistical techniques for training set. The third approach is Nested Combining Classifiers (NCC that based on adding the outputs combination using product rule (PR1) to the inputs combination using neural network (NN1), which presented an additional information that the improvement of network performance. In (NN+LR) approach the $\mathrm{NN}$ is the first combination classifier, while in $(\mathrm{LR}+\mathrm{NN})$ logistic regression is the first combination classifier and it doesn't need a specified condition in the medical data.

\section{1- Acquiring the Data}

The applied area of this study was Extracorporeal Shock Wave Lithotripsy (ESWL) for Renal Stones, where the renal stones represent the most important disorders which affect the Urinary tract. When we discover the present of a stone, it is treated by three ways:

- ESWL.

- Surgery.

- Ureteroscopy or Percutaneous Nephrolithotomy (PNL).

We use ESWL when the length of stone is less than $30 \mathrm{~mm}$ and the outcome of treatment is one of two:

- The patient becomes free from any fragments of stone.

- The patient becomes not free.

Where $y$ is the dependent variable for the outputs of ESWL, Thus:

$$
y= \begin{cases}1 & \text { if } \quad \text { the patient is free } \\ 0 & \text { if } \quad \text { the patient is not free }\end{cases}
$$

The model contains the following twelve independent variables, they are:

1- Age $\left(x_{1}\right)$.

2- Stones

Number $\left(x_{7}\right)$.

3- $\operatorname{Sex}\left(x_{2}\right)$.

4- Stones

Length $\left(x_{8}\right)$.

5- Morphology $\left(x_{3}\right)$. 6- Stones

Site $\left(x_{9}\right)$.

7- Anatomy $\left(x_{4}\right)$. 8- Stones

Nature $\left(x_{10}\right)$.

9- Use of JJ Stent $\left(x_{5}\right)$. 10- Side

$\left(x_{11}\right)$.

11- Solitary $\left(x_{6}\right)$.

12- Stones

Opacity $\left(x_{12}\right)$.

The data has been taken from real cases at "Al Karama Teaching Hospital" and "Al Yarmuk General Teaching Hospital", where the patents data has been put in database. Figure 4 shows the samples of ESWL image and data includes patient information. 

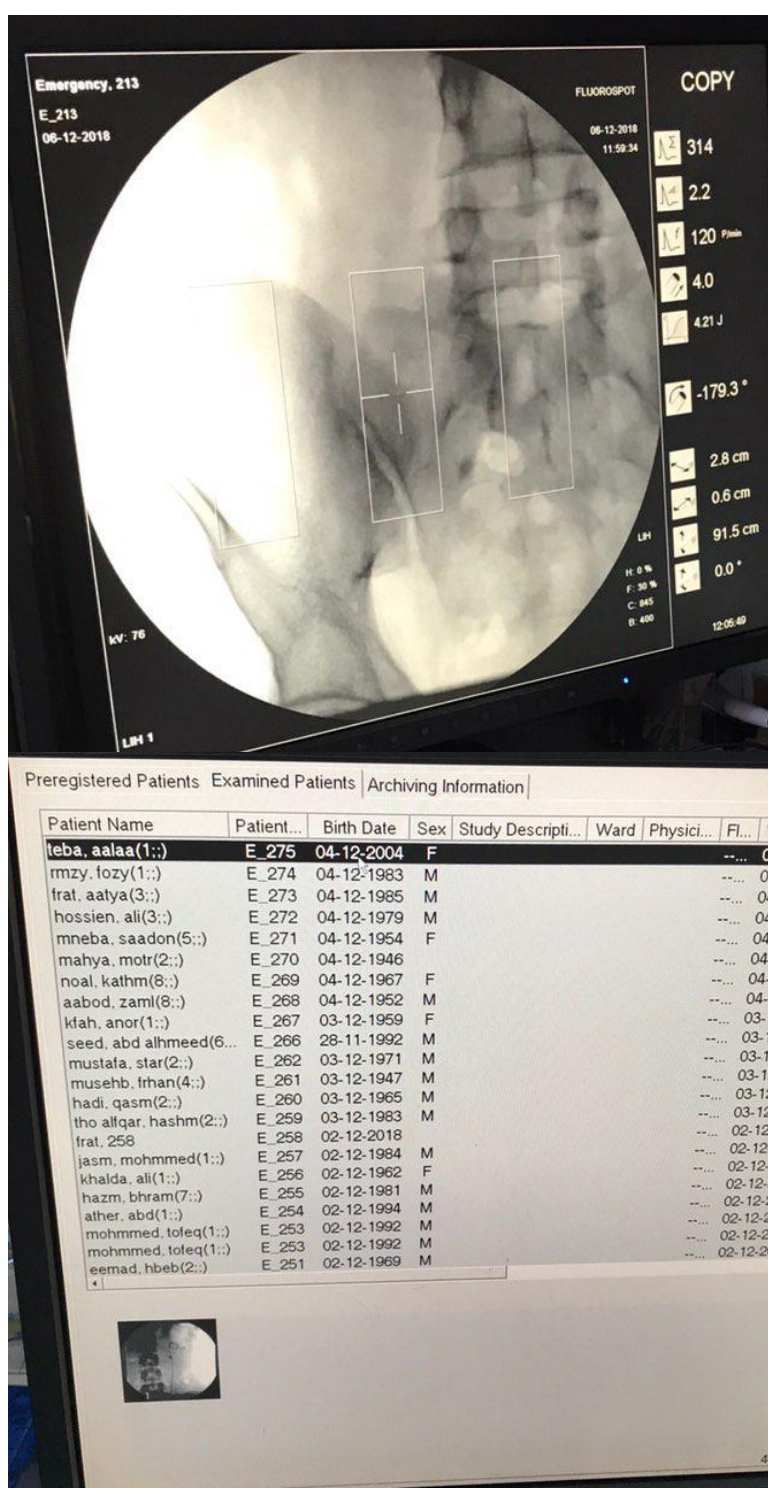

Figure 3: ESR Devise. Left, the kidney image

and stone detected. On the right, the patient information

\subsection{Combining Using Product Rule (PR1)}

The product rule method is used to combine the Logistic Regression results which present the probability of belonging to class with Neural Networks results which represent the probability of belonging to class. The application of this method is used the testing sample and training sample.

\subsection{Combining Using Neural Network (NN1)}

In this method we are adding outputs of the statistical techniques for training set which present belonging the probabilities for classes and these probabilities are one of the inputs Neural Network. So, we will keep twelve Hidden Neurons to ensure that the improvement of improvement of Network performance due to the additional information which is presented in the outputs of statistical technique.

\subsection{Nested Combining Classifiers (NCC):}

In this study, we provide a new technique of combination in classification area, hoping to increase the accuracy of classification, which named Nested Combining Classifier in which we combine the results of combination using Neural Network and the results of combination using Product Rule, i.e. we combine between two results of combination. Methods of combining classifiers of the three types are shown in Figure 2.

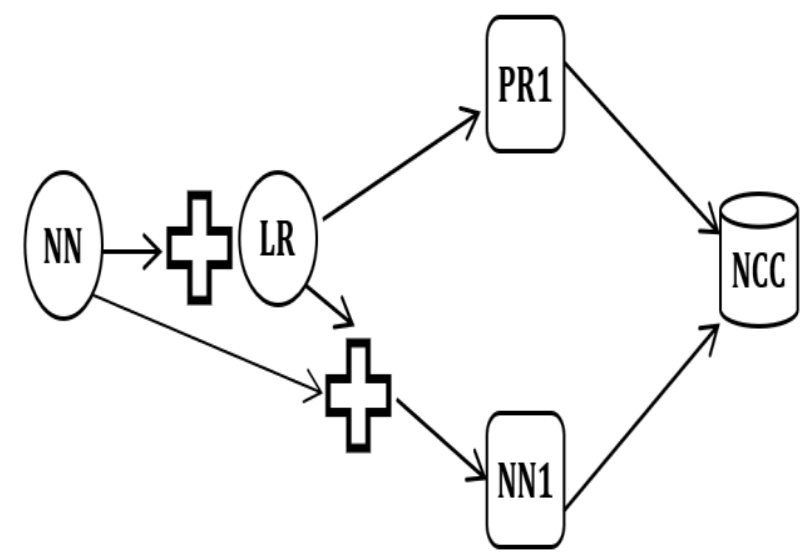

Figure 4: Methods of combining classifiers of the three types

Where: NN: is Neural Network, LR: is Logistic Regression, PR1: is combining using Product Rule, NN1: is combining using Neural Network, and NCC: is Nested Combined Classifier.

In the Nested Combined Classifier (NNC) method we combine between PR1 and NN1. To explain this method, we add the results of the product Rule to the inputs of the neural network (NN1), i.e. combined between two results of combination. In this section, we will explore the method that used to evaluate classification models. 


\section{4- Results}

In this section we test the three approaches for predicate the success of SWL in treatment the patient, where the samples had been taken from 3225 actual cases that had been treated at Urology and Nephrology center of Iraq ("Al Karama Teaching Hospital" and "Al Yarmuk General Teaching Hospital"). We selected 2800 cases for training and 425 select to be a nontrained cases. For trained data, there are 538 not free cases (failed to fee stone) and 2262 free cases (Successes in free stone). For no- trained, there are 47 not free cases (failed to fee stone) and 378 free cases (Successes in free stone). The results from three cases have been compared to actual treatment results of SWL for trained and nontrained cases and compered the results of three models.

\section{4-1 Results of Combination Using Product Rule (PR1):}

The results of predicate SWL by PR1 are shown in Table 1, which shows the results for classification by using PR for each sample (testing \& training).

Table 1: The results for classification by using

\begin{tabular}{|c|c|c|c|c|c|c|}
\hline \multirow{4}{*}{ Observation } & \multicolumn{6}{|c|}{ Predicted } \\
\hline & \multicolumn{3}{|c|}{$\begin{array}{c}\text { Trained Cases (2800) } \\
\text { Not Free }(538), \text { Free } \\
(2262)\end{array}$} & \multicolumn{3}{|c|}{$\begin{array}{c}\text { Non-Trained Cases } \\
\text { (425) } \\
\text { Not Free (47), Free } \\
(378) \\
\end{array}$} \\
\hline & \multicolumn{2}{|c|}{$\mathbf{y}$} & \multirow{2}{*}{$\begin{array}{l}\text { Percent } \\
\text { age } \\
\text { Correc } \\
\text { t } \\
\end{array}$} & \multicolumn{2}{|c|}{$\mathbf{y}$} & \multirow{2}{*}{$\begin{array}{r}\text { Percent } \\
\text { age } \\
\text { Correct }\end{array}$} \\
\hline & $\begin{array}{l}\text { Not } \\
\text { free }\end{array}$ & Free & & $\begin{array}{l}\text { Not } \\
\text { free }\end{array}$ & $\begin{array}{l}\text { Fr } \\
\text { ee }\end{array}$ & \\
\hline y Not free & 453 & 85 & $84.2 \%$ & 34 & 13 & $72.34 \%$ \\
\hline y Free & 319 & 1943 & $85.9 \%$ & 110 & $\begin{array}{c}26 \\
8\end{array}$ & $70.9 \%$ \\
\hline $\begin{array}{l}\text { Overall } \\
\text { Percentage }\end{array}$ & 459 & 2395 & $85.31 \%$ & ד4 & $\begin{array}{c}28 \\
9\end{array}$ & $68 \%$ \\
\hline
\end{tabular}

PR1 for testing and training sample

$[$ ROC (Training Set) $=0.85$, ROC $($ Testing Set $)=0.72]$

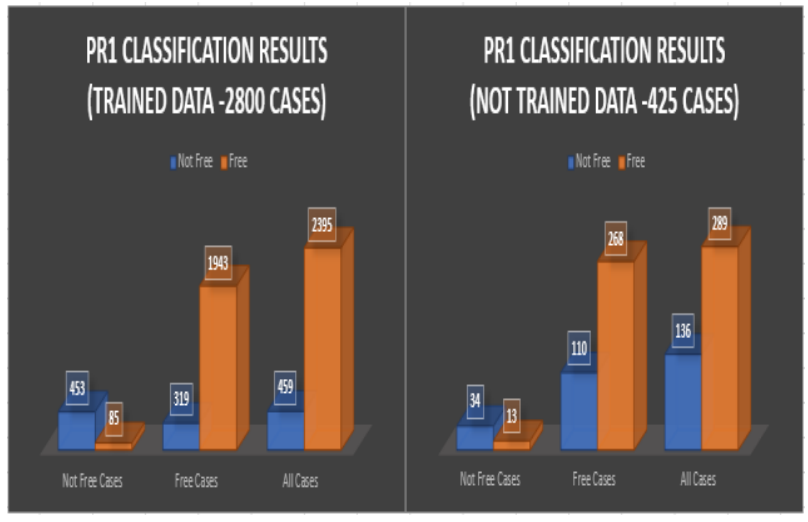

(a)

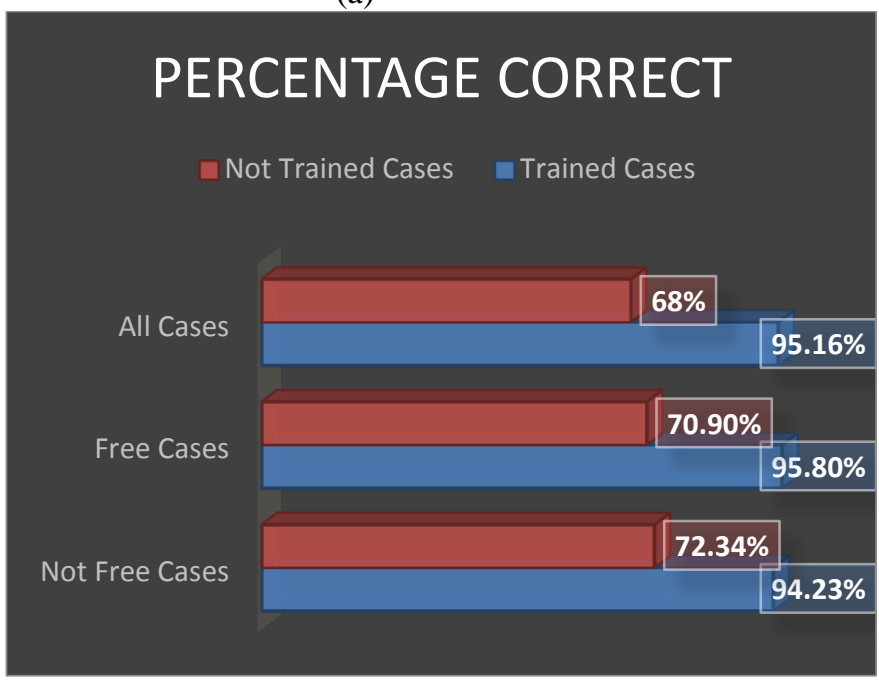

(b)

Figure 5: PR1 Classification results. (a) Classification Results for trained and not trained cases, (b) Percentage correct of classification

As shown from table 1 and figure 5 the system successes in detect of 453 non-free cases from 538 non-free cases that used in train which got $(84.2 \%)$ accuracy, and for not trained data the system successes in detect of 34 non-free cases from 47 non-free cases that used in train which got $(72.34 \%)$ accuracy. For free stone cases, the system successes in detect of 1843 free cases from 2262 free cases that used in train which got $(81.47 \%)$ accuracy, and for not trained data the system successes in detect of 268 free cases from 378 free cases that used in train which got (70.9\%) accuracy

\section{4-2 Results Combination Using Neural Network (NN1):}

The result of predicate SWL by NN1 are shown in Table 2, which shows the results for classification by using $\mathrm{NN}$ for each sample (testing \& training). 
Table 2: The results for classification by using NN1 for testing and training sample

\begin{tabular}{|c|c|c|c|c|c|c|}
\hline \multirow{4}{*}{ Observation } & \multicolumn{6}{|c|}{ Predicted } \\
\hline & \multicolumn{3}{|c|}{$\begin{array}{c}\text { Trained Cases }(\mathbf{2 8 0 0}) \\
\text { Not Free }(538), \text { Free }(2262)\end{array}$} & \multicolumn{3}{|c|}{$\begin{array}{c}\begin{array}{c}\text { Non-Trained Cases } \\
(\mathbf{4 2 5})\end{array} \\
\text { Not Free (47), Free (378) }\end{array}$} \\
\hline & \multicolumn{2}{|c|}{$\mathbf{y}$} & \multirow{2}{*}{$\begin{array}{l}\text { Percent } \\
\text { age } \\
\text { Correct }\end{array}$} & \multicolumn{2}{|c|}{$\mathbf{y}$} & \multirow{2}{*}{$\begin{array}{r}\text { Percent } \\
\text { age } \\
\text { Correct }\end{array}$} \\
\hline & $\begin{array}{l}\text { Not } \\
\text { free }\end{array}$ & Free & & $\begin{array}{l}\text { Not } \\
\text { free }\end{array}$ & $\begin{array}{c}\text { Fre } \\
\text { e }\end{array}$ & \\
\hline y Not free & 507 & 31 & $94.23 \%$ & 38 & 9 & $80.85 \%$ \\
\hline y Free & 95 & 2167 & $95.8 \%$ & 56 & 322 & $85.18 \%$ \\
\hline $\begin{array}{l}\text { Overall } \\
\text { Percentage }\end{array}$ & 512 & 2288 & $95.16 \%$ & 39 & 386 & $82.97 \%$ \\
\hline
\end{tabular}

$[$ ROC (Training Set) $=0.96$, ROC $($ Testing Set $)=0.81]$

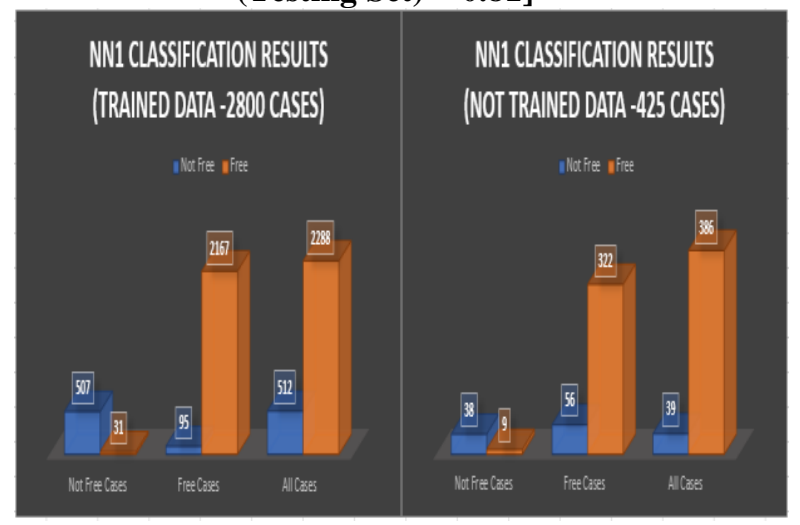

(a)

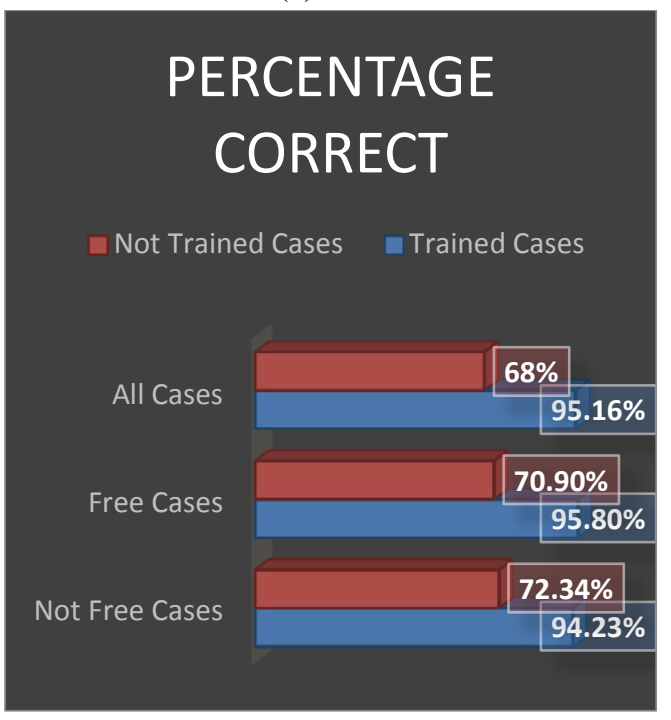

(b)

Figure 6: NN1 Classification results. (a) Classification Results for trained and not trained cases, (b) Percentage correct of classification
As shown from table 2 and figure 6 the system successes in detect of 507 non-free cases from 538 non-free cases that used in train which got $(94.23 \%)$ accuracy, and for not trained data the system successes in detect of 38 non-free cases from 47 non-free cases that used in train which got $(80.85 \%)$ accuracy. For free stone cases, the system successes in detect of 2167 free cases from 2262 free cases that used in train which got $(95.16 \%)$ accuracy, and for not trained data the system successes in detect of 322 free cases from 387 free cases that used in train which got $(85.18 \%)$ accuracy.

\section{4-3 Results of the Nested Combined Classifier} (NCC):

The Nested Combined Classifier method is carried out by adding the outputs combination using Product Rule (PR1) to the inputs combination using Neural Network (NN1), which present additional information that the improvement of network performance.

Table 3: The results for classification by using

\begin{tabular}{|c|c|c|c|c|c|c|}
\hline \multirow{4}{*}{ Observation } & \multicolumn{6}{|c|}{ Predicted } \\
\hline & \multicolumn{3}{|c|}{$\begin{array}{c}\text { Trained Cases (2800) } \\
\text { Not Free (538), Free (2262) }\end{array}$} & \multicolumn{3}{|c|}{$\begin{array}{l}\text { Non-Trained Cases (425) } \\
\text { Not Free (47), Free (378) }\end{array}$} \\
\hline & \multicolumn{2}{|c|}{$\mathbf{y}$} & \multirow{2}{*}{$\begin{array}{c}\text { Percenta } \\
\text { ge } \\
\text { Correct }\end{array}$} & \multicolumn{2}{|c|}{$\mathbf{y}$} & \multirow{2}{*}{$\begin{array}{r}\text { Percenta } \\
\text { ge } \\
\text { Correct }\end{array}$} \\
\hline & $\begin{array}{l}\text { Not } \\
\text { free }\end{array}$ & Free & & $\begin{array}{l}\text { Not } \\
\text { free }\end{array}$ & $\begin{array}{c}\text { Fre } \\
\text { e }\end{array}$ & \\
\hline y Not free & 522 & 16 & $97.02 \%$ & 41 & 9 & $87.23 \%$ \\
\hline y Free & 24 & 2167 & $98.93 \%$ & 37 & 341 & $90.21 \%$ \\
\hline Overall Percentage & 527 & 2273 & $97.95 \%$ & 43 & 382 & $91.5 \%$ \\
\hline
\end{tabular}

[ROC (Training Set) $=0.977$, ROC $($ Testing Set $)=0.875]$

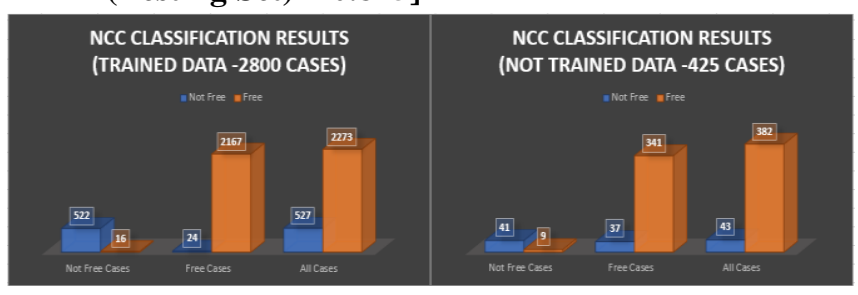

(a) 
Journal of AL-Qadisiyah for computer science and mathematics $\quad$ Vol.11 No.1 Year 2019

ISSN (Print): 2074 - $0204 \quad$ ISSN (Online): 2521 - 3504

Samera.Sh/Lubab.A/ Sukaina .Sh

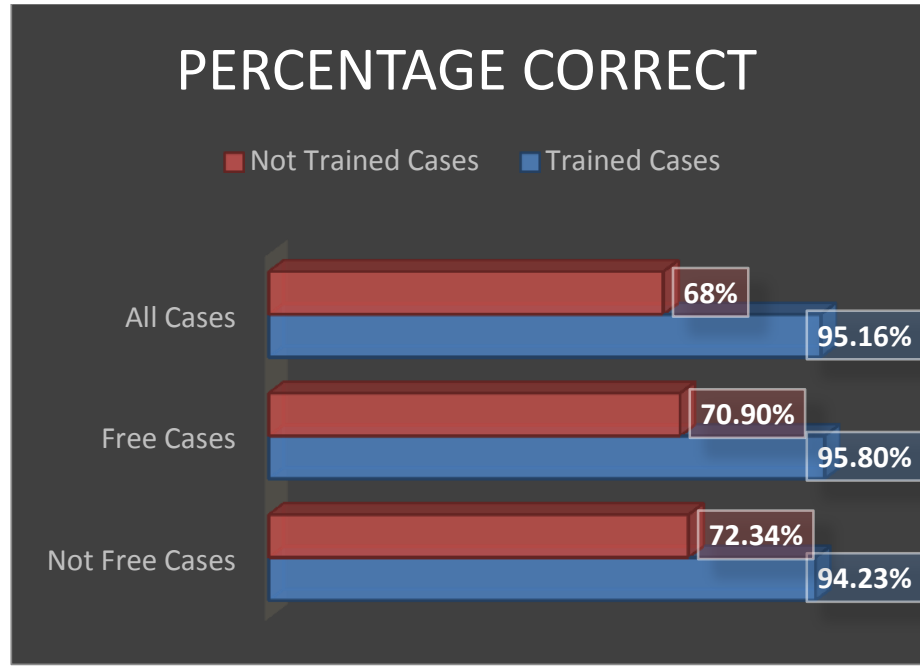

(b)

Figure 7: PR1 Classification results. (a) Classification Results for trained and not trained cases, (b) Percentage correct of classification

As shown from table 3 and figure 7 the system successes in detect of 522 non-free cases from 538 non-free cases that used in train which got (97.02\%) accuracy, and for not trained data the system successes in detect of 41 non-free cases from 47 non-free cases that used in train which got $(87.23 \%)$ accuracy. For free stone cases, the system successes in detect of 2167 free cases from 2262 free cases that used in train which got $(98.93 \%)$ accuracy, and for not trained data the system successes in detect of 341 free cases from 378 free cases that used in train which got (90.21\%) accuracy

\section{4-4 Comparison between the Results:}

The percentage results of the three classification techniques are displayed is table 4 as in follows:

\begin{tabular}{|c|c|c|c|c|}
\hline \multirow{2}{*}{$\begin{array}{c}\text { Techn } \\
\text { ique }\end{array}$} & \multicolumn{2}{|c|}{ Training Sample } & \multicolumn{2}{c|}{ Testing Sample } \\
\cline { 2 - 5 } & $\begin{array}{c}\text { Overall } \\
\text { Percentage }\end{array}$ & $\begin{array}{c}\text { The Area } \\
\text { under ROC } \\
\text { Curve }\end{array}$ & $\begin{array}{c}\text { Overall } \\
\text { Percentage }\end{array}$ & $\begin{array}{c}\text { The Area } \\
\text { under ROC } \\
\text { Curve }\end{array}$ \\
\hline PR1 & $85.31 \%$ & $\mathbf{0 . 8 5}$ & $68.00 \%$ & $\mathbf{0 . 7 2}$ \\
\hline NN1 & $95.16 \%$ & $\mathbf{0 . 9 6}$ & $82.97 \%$ & $\mathbf{0 . 8 1}$ \\
\hline NCC & $97.95 \%$ & $\mathbf{0 . 9 7}$ & $91.50 \%$ & $\mathbf{0 . 8 7}$ \\
\hline
\end{tabular}

Table 4: Summary of the results for $\mathrm{C}$ techniques

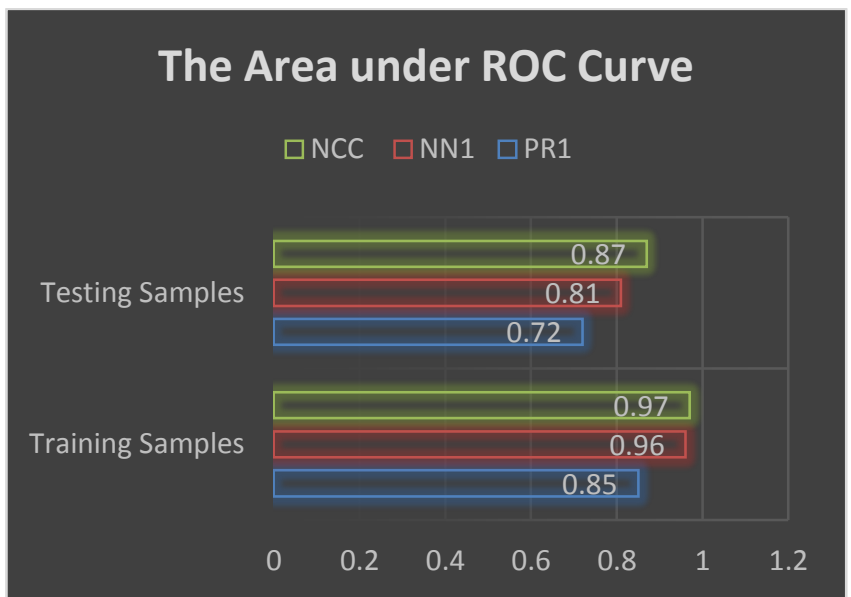

Overall Percentage

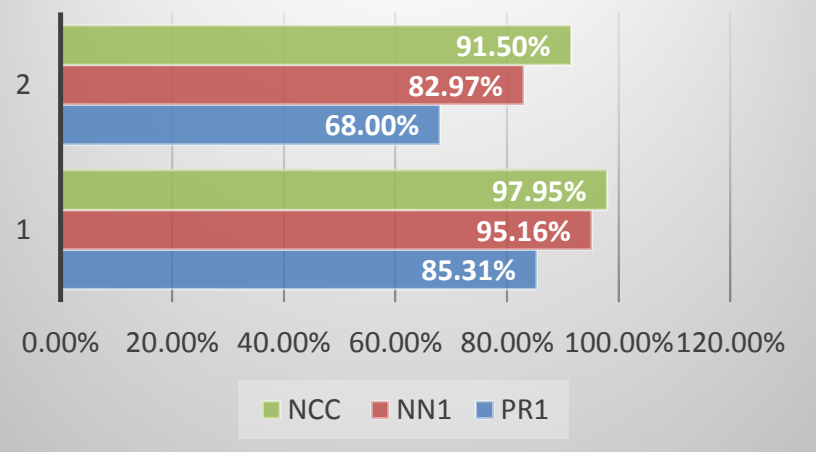

Figure 8: NN Classification results. (a)

Classification Results for trained and not trained cases, (b) Percentage correct of classification

Table 4 and figure 8 shows summary of the results the overall percentage and the area under ROC curve for classification techniques of each testing sample and training sample. We compare between three techniques which are PR1, NN1, and NCC. As shown from table 4 the best technique for classification is the Nested Combined Classifier (NCC), where the overall percentage of the classification for training sample with NCC is $(97.95 \%)$ which is higher than $(85.31 \%)$ of PR1 and $(95.16 \%)$ of NN1. Also, its shows that the overall percentage for testing sample of NCC is $(91.5 \%)$ which is higher than the PR1 (68\%) and NN1 (82.97\%). The area under ROC curve of the NCC for training sample is (0.97) which is higher than (0.85) of PR1 and (0.96) of NN1, and the area under ROC curve of NCC for testing sample is (0.87) which is also higher than PR1 and NN1 which are $(0.72$ and 0.81 ) respectively. 
By compared with previous works of [6] and [8], the results achieved higher accuracy than [6] in both $\mathrm{NN}$ and NCC where the accuracy of training result got about $22 \%$ accuracy improvement in training data detection and about $10 \%$ accuracy improvement in tested data. By compared with [8] the both got close accuracy result in detection of trained data where NCC got $\sim 98 \%$ while their method achieved relatively higher accuracy of $99.25 \%$, but for non-trained (tested) samples the proposed method (NCC) has achieved more accuracy reach to $91.5 \%$ which $3 \%$ higher accuracy than their method that achieved $88.7 \%$.

\section{Conclusions}

Machine learning Techniques, such as the ANN, has been used widely in the medical field, as computer generated algorithms, that assist healthcare officials in clinical making decisions. One of medical application is to predicate the probability of success in desired treatment. For that purpose, three models to predicate the ability of ESWR to remove stone from kidney has been presented and tested, which based on used three techniques of mixing classifiers, PR, NN and NCC. We have been designed and develop a theoretical framework for combined classifiers and study the challenge of combining classifiers that uses different representations of the patterns for being classified. The testing shows that numerous existing schemes is often considered as unique cases of compound classification in which every pattern representations being used jointly to make a decision. The results show that the best technique for classification is the Nested Combined Classifier (NCC) when compared with other combination classifiers (NN and PR). Also, it should be emphasized that the analysis of result is dependent on a single experimentation for a single dataset. Thus, the conclusions can be summarized as in follows:

- Combining classifiers trained on a variety of feature sets is beneficial. Mostly in cases where these feature set probabilities are estimated via the classifier. On the other hand, combining the different classifiers trained with the same classifier might improve but is usually much less useful.
- The separated feature sets work well when used independently. Difficult datasets will not be thrown away; they include significant information! Using randomly selected feature sets seems to provide excellent results in our study.

- A resealing of all features sets to unit variance maybe enhance the accuracy of number of classifiers.

\section{References:}

[1] Torricelli, F.C.M.; Danilovic, A.; Vicentini, F.C.; Marchini, G.S.; Srougi, M.; Mazzucchi E. 2015. Extracorporeal Shock Wave Lithotripsy in the Treatment of Renal and Ureteral Stones. Rev Assoc Med Bras; 61(1), pp.65-71, DOI: 10.1590/18069282.61.01.065.

[2] Trew, J.; Cornfield, J. 2017. A Comparison of Treatment Modalities in Renal and Ureteral Calculi. Open Journal of Urology, 7, pp.47-53, DOI: 10.4236/oju.2017.73007.

[3] Augustin, H. 2007. Prediction of StoneFree Rate after ESWL. European Association of Urology, Elsevier B.V., 5(2), pp.318-320, DOI: 10.1016/j.eururo.2007.03.058.

[4] Ayer, T.; Chhatwal, J.; Alagoz, O.; Kahn, C.E.; Woods, R.W.; Burnside, E.S. 2010. Comparison of Logistic Regression and Artificial Neural Network Models in Breast Cancer Risk Estimation. Radiographics. 30(1), pp.13-22, DOI: 10.1148/rg.301095057.

[5] Lisboa, P.J. and Taktak A.F. 2006. The use of artificial neural networks in decision support in cancer: a systematic review. Neural Networks, Elsevier Ltd., Vol.19, Issue 4, pp.408-415.

[6] Hamid, A.; Dwivedi, U.S.; Singh, T.N.; Gopi Kishore, M.; Mahmood, M.; Singh, H.; Tandon, V.; Singh, P.B. 2003. Artificial Neural Networks in Predicting Optimum Renal Stone Fragmentation by Extracorporeal Shock Wave Lithotripsy: A Preliminary Study. BJU International 91, pp.821-824, DOI: 10.1046/j.1464410X.2003.04230. x. 
[7] Goyal N. K., Kumar A., Trivedi S., Dwivedi U. S., Singh T. N., and Singh P. B. 2010. A Comparative Study of Artificial Neural Network and Multivariate Regression Analysis to Analyze Optimum Renal Stone Fragmentation by Extracorporeal Shock Wave Lithotripsy. Saudi Center for Organ Transplantation., Saudi J Kidney Dis Transplant; 21(6) pp.1073-1080.

[8] Seckiner I., Seckiner S., Sen H., Bayrak O., Dogan K., and Erturhan S. 2016. A Neural Network - Based Algorithm for Predicting Stone - Free Status After ESWL Therapy. International Brazilian Journal of Urology (IBJU), Vol.43 (6), pp.1110-1114, DOI: 10.1590/S16775538.IBJU.2016.0630.
[9] Hassan, M.; Butt, M.A.; Baba, M.Z. 2017. Logistic Regression Versus Neural Networks: The Best Accuracy in Prediction of Diabetes Disease.

[10] Koç, A. A.; Yenia, Ö. 2013. A Comparative Study of Artificial Neural Networks and Logistic Regression for Classification of Marketing Campaign Results. Mathematical and Computational Applications, Vol.18, No 3, pp. 392-398.

[11] Arana, E.; Delicado, P.; MartíBonmatí, L. 1999. Validation procedures in radiological diagnostic models. Neural network and logistic regression. Investigative Radiology, Department of Radiology. Hospital Casa de Salud. Manuel Candela.

\section{توقع قدرة تقنية تَفَتِتُ الحَصاةِ بمَوجات صادِِمَةٍ مِنْ خارِجِ الجسم (ESWL) على علاج

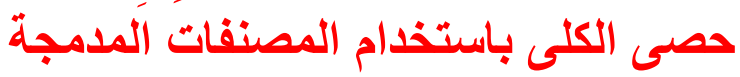

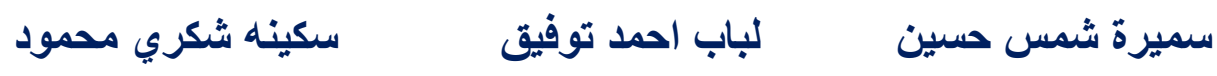

قسم علوم الحاسبات، كلية التربية للعلوم الصرفة، جامعة بغداد

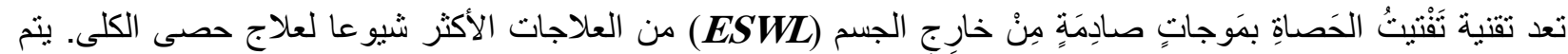

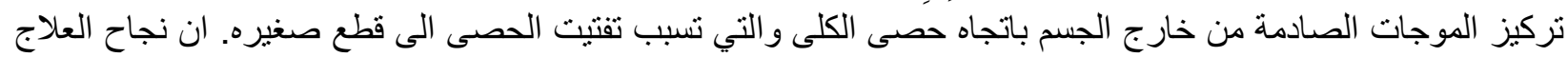
بتقنية (ESWL) يعتمد على عدة عوامل مثل: العمر، الجنس، حجم الحصى، عدد الحصى وغير ها. وبالتالي، فإن التنبؤ

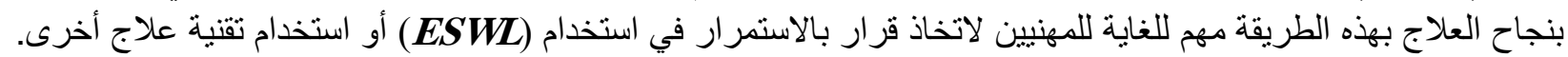

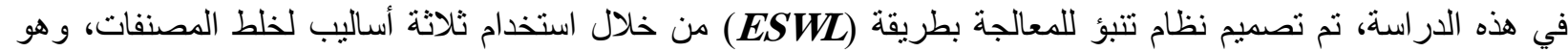

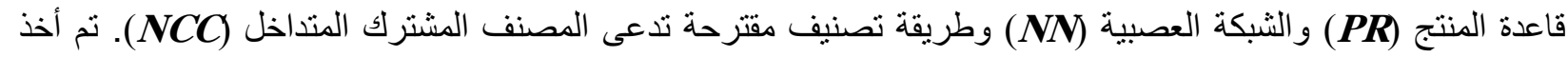

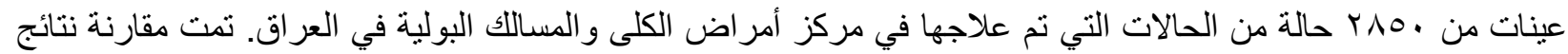

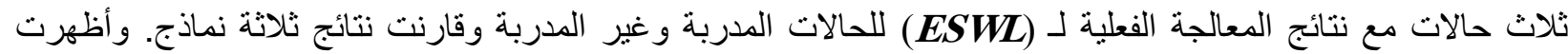

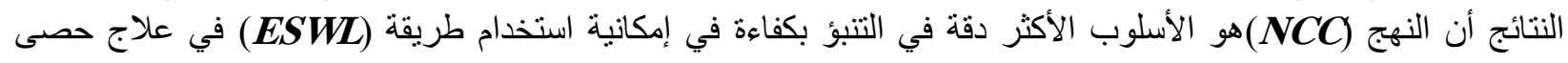

PR،ANN، الكلمات المفتاحية: تَفْتبتُ الحَصاةِ بمَوجاتٍ صادِمَةٍة ، قاعدة المنتج، الثبكة العصبية 